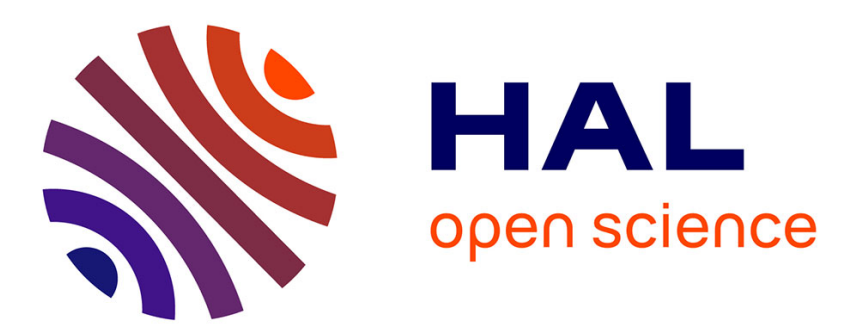

\title{
Impact of visual angle on attention deployment and robustness of visual saliency models in videos: From SD to UHD
}

\author{
Toinon Vigier, Matthieu Perreira da Silva, Patrick Le Callet
}

\section{- To cite this version:}

Toinon Vigier, Matthieu Perreira da Silva, Patrick Le Callet. Impact of visual angle on attention deployment and robustness of visual saliency models in videos: From SD to UHD. IEEE International Conference on Image Processing 2016 (ICIP), Sep 2016, Phoenix, United States. pp.689 - 693, 10.1109/ICIP.2016.7532445 . hal-01438356

\section{HAL Id: hal-01438356 https://hal.science/hal-01438356}

Submitted on 17 Jan 2017

HAL is a multi-disciplinary open access archive for the deposit and dissemination of scientific research documents, whether they are published or not. The documents may come from teaching and research institutions in France or abroad, or from public or private research centers.
L'archive ouverte pluridisciplinaire HAL, est destinée au dépôt et à la diffusion de documents scientifiques de niveau recherche, publiés ou non, émanant des établissements d'enseignement et de recherche français ou étrangers, des laboratoires publics ou privés. 


\title{
IMPACT OF VISUAL ANGLE ON ATTENTION DEPLOYMENT AND ROBUSTNESS OF VISUAL SALIENCY MODELS IN VIDEOS: FROM SD TO UHD
}

\author{
Toinon Vigier, Matthieu Perreira Da Silva, Patrick Le Callet \\ IRCCyN CNRS UMR 6597, Université de Nantes, France
}

\begin{abstract}
The emergence of UHD video format induces larger screens and involves a wider stimulated visual angle. Therefore, its effect on visual attention can be questioned since it can impact quality assessment, metrics but also the whole chain of video processing and creation. Moreover, changes in visual attention from different viewing conditions challenge visual attention models. In this paper, we present a comparative study of visual attention and viewing behavior on three video datasets in SD, HD and UHD conditions. Then, we propose and assess an improvement for video visual attention models by applying a stimulated visual angle dependent center model.
\end{abstract}

Index Terms - Visual attention, video, UHD, visual saliency model, eye tracking.

\section{INTRODUCTION}

UHD TV standard defines new video technologies as an increasing resolution from $\mathrm{HD}(1920 \times 1080)$ to $4 \mathrm{~K}(3840 \times 2160)$ or $8 \mathrm{~K}(7680 \times 4320)$. Thus, the emergence of UHD potentially provides a better immersion of the user thanks to a wider visual angle with appropriate larger screens [1]. Indeed, ITU defines the optimal viewing distance as the distance at which scanning lines just cannot be perceived with visual acuity of 1'. It is thus set to $6 \mathrm{H}$ for $\mathrm{SD}, 3 \mathrm{H}$ for $\mathrm{HD}$ and $1.5 \mathrm{H}$ for UHD where $\mathrm{H}$ is the height of the screen [2]. Figure 1 shows the increase of stimulated visual angle along with a better resolution.

Hence, two questions can be raised:

1. Does stimulated visual angle impact viewing behavior and strategies?

2. How can visual angle be taken into account to improve visual attention prediction?

Recent studies highlight the effect of transition from HD to UHD on visual attention $[3,4,5]$. By comparing visual attention in UHD and HD static images, Nemoto et al. pointed out that viewing strategy and visual attention are significantly different in these two cases: UHD images can grab the focus of attention more than HD images. Moreover, several models of visual saliency were compared in HD and UHD scenarios, showing a reduction of model performance in UHD [4].
However, viewing behavior in video differs from static images, preventing the straightforward use of these observations for dynamic content. To our knowledge, the first UHD video saliency database was published in [5]. These data come with a comparison of viewing behavior in UHD and HD scenarios. It was especially shown that viewer attention was more focused on the center of the screen in HD context. Nevertheless, no statistical analysis was provided and the performance of visual saliency models in UHD videos was not tackled.

In this paper, we propose to evaluate the impact of stimulated visual angle on visual attention deployment in videos and on the robustness of visual attention models. First, we assess visual patterns by comparing gaze data of three eye tracking datasets in SD, HD and UHD conditions. Then, we present and assess an improvement of video visual attention models by applying a visual angle dependent center model.

\section{MATERIALS AND METHODS}

In this section, we present SD, HD and UHD eye tracking datasets and metrics used in this comparative study.

\subsection{Description of datasets}

The used datasets are IVC_SD [6], SAVAM [7] and a new UHD dataset, IVC_UHD. All these three datasets fulfill the following criteria: public availability, description of the viewing conditions, free-looking approach, no soundtrack, and most importantly, respect of the ITU viewing distance recommendations [8]. Because there is no public SD, HD and UHD eye tracking datasets sharing exactly the same content, we chose datasets with similar content, i.e. professional videos often used for subjective video quality assessment.

IVC_SD is an eye tracking dataset of SD videos compressed in good quality with HEVC. Some of the 31 sequences were presented in two versions: with and without transmission errors. We only use the transmission error-free version.

SAVAM is an HD visual attention dataset on high quality video sequences. Some observers viewed the sequences twice in reverse order. In this case, we only use the first viewing to avoid memory effects. 


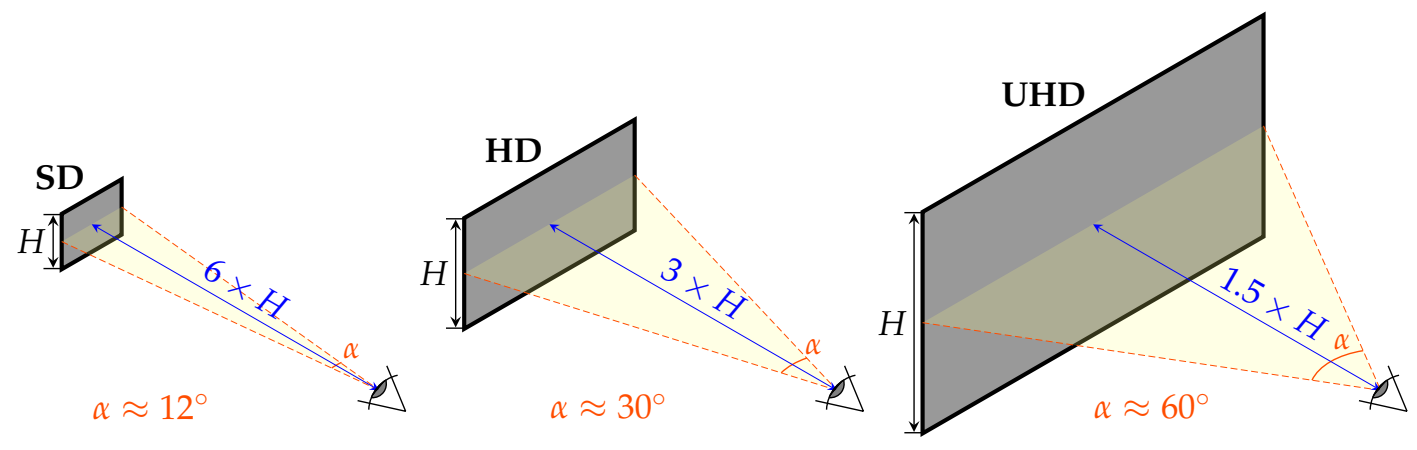

Fig. 1. The increase of stimulated visual angle from SD to UHD.

IVC_UHD is a new UHD dataset produced in IRCCyN laboratory on 78 reference UHD video sequences including a large variety of scenes. Because of the wider visual angle in UHD, observers can need to move their head and eye tracking systems may not be accurate enough at the edges of the screen. Therefore, we developed a new eye tracking setup EHT (Eye Head Tracking) that is a combination of the mobile SMI eye tracking glasses and of the head tracker OptiTrack ARENA. The experiment was conducted in a test environment set as a standard subjective quality test condition according to ITU-R BT.500 [9]. This new UHD dataset will be available at http://ivc.univ-nantes.fr/.

The detailed description for each dataset is provided in Table 1.

\begin{tabular}{lccc}
\hline & IVC_SD & SAVAM & IVC_UHD \\
\hline Resolution & $720 \times 576$ & $192 \times 1080$ & $3840 \times 2160$ \\
Width (mm) & 287.5 & 531.6 & 1422.2 \\
Distance (mm) & 1380 & 1000 & 1200 \\
$\begin{array}{l}\text { Distance/Height } \\
\text { Horizontal }\end{array}$ & $\mathbf{1 1 . 8 9}$ & 3.3 & 1.5 \\
visual angle ( & $\left.{ }^{\circ}\right)$ & $\mathbf{2 9 . 7 7}$ & $\mathbf{6 1 . 3 0}$ \\
Nb of observers & 49 & 48 & 37 \\
$\begin{array}{l}\text { Nb of sources } \\
\text { Sequence length }\end{array}$ & 31 & 32 & 78 \\
(sec) & 9 & 18 & 12 \\
\hline
\end{tabular}

Table 1. Description of eye tracking datasets.

\subsection{Saliency maps}

We directly compute saliency maps on gaze positions rather than fixations as in [10]. Then, gaze points are convolved with a bidimensional gaussian function with $\sigma=1^{\circ}$ of visual angle as recommended in [11]. It corresponds to a full width at half maximum (FWHM) of $2.2^{\circ}$ which is approximately the size of the fovea. Saliency maps are computed according to the proposed methodology for each video of each dataset.

\subsection{Metrics}

\subsubsection{Dispersion}

To evaluate the impact of stimulated visual angle on visual attention, we analyze the dispersion of gaze data through two metrics computed for each video of datasets: the mean and the standard deviation of the distances in degree of visual angle between gaze points and the center of the screen over all sequence video frames. In the following, we denote the mean of distances for one video sequence as $\overline{d_{\text {seq }}}$ and the standard deviation of distances for one video sequence as $\sigma_{d_{\mathrm{seq}}}$.

\subsubsection{Comparison with center models}

Center bias is a well know phenomenon in visual attention deployment corresponding to the the tendency to gaze mostly at the center of the visual content. This bias would arise from different causes as motor bias, viewing strategy or video content $[10,12,13]$. To evaluate the distribution of gaze points around the center of the video, we compare the experimental saliency maps with center models thanks to Pearson correlation based measures $(\mathrm{Cp})$ and Kullback-Liebler divergence (KLD) as recommended in [11]. Here, center models correspond to anisotropic 2D gaussian centered in the map. The ratio of the gaussian preserves the ratio of the map. The width is expressed in visual angle degrees and it represents the FWHM of the gaussian. Figure 2 depicts a $10^{\circ}$ center model in SD, HD and UHD viewing conditions.

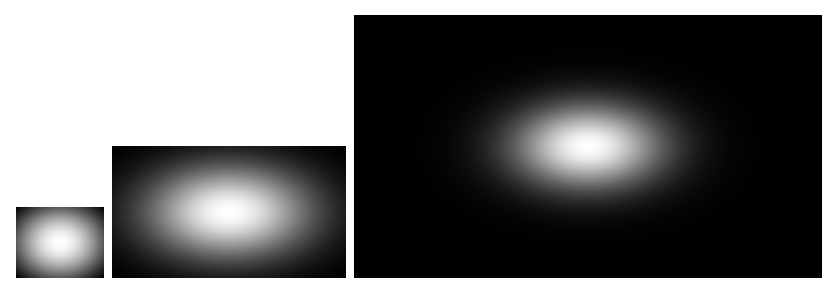

Fig. 2. Center models with width $=10^{\circ}$ in SD, HD and UHD. 


\section{IMPACT OF STIMULATED VISUAL ANGLE ON GAZE DATA DISTRIBUTION}

In this section we compare and discuss results obtained on the different datasets with the metrics presented in the previous section. We refer to horizontal stimulated visual angle in degree as $\alpha$.

\subsection{Dispersion}

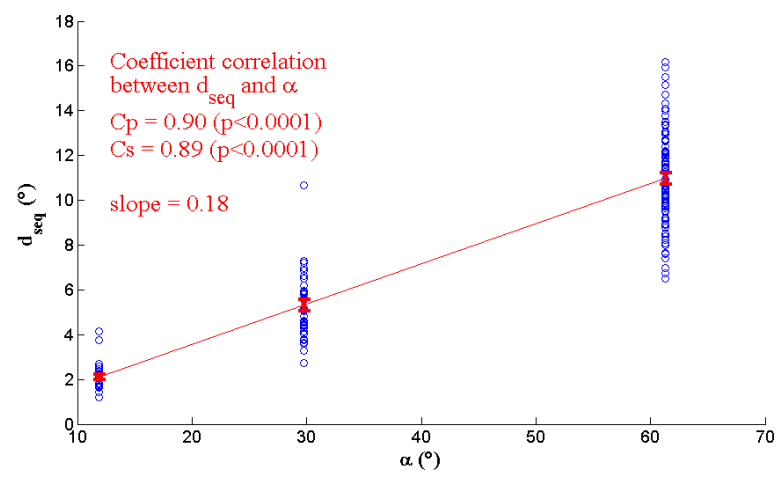

Fig. 3. Impact of visual angle on $\overline{d_{\text {seq }}}$.

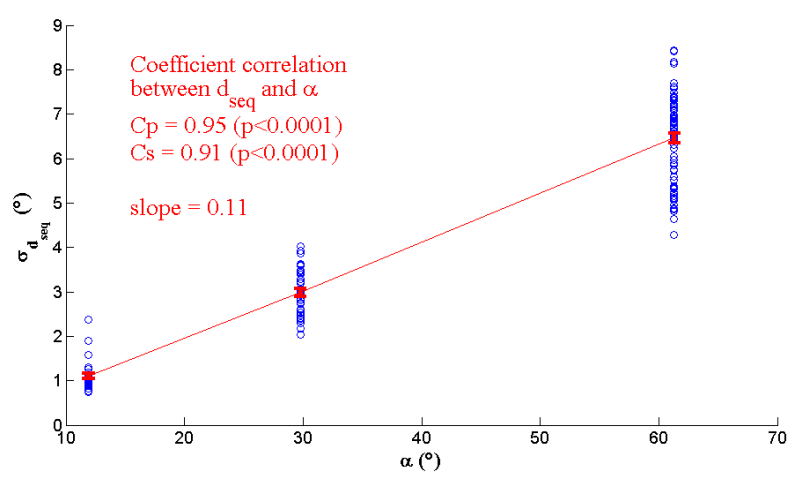

Fig. 4. Impact of visual angle on $\sigma_{d_{\text {seq }}}$.

Figure 3 shows that $\overline{d_{\text {seq }}}$ linearly increases along with the visual angle. The ratio between $\overline{d_{\text {seq }}}$ and $\alpha$ remains nearly constant around 0.18 . A Kruskal-Wallis test validates that $\overline{d_{\text {seq }}} / \alpha$ is not significantly different between datasets $(\mathrm{p}=0.66)$. Figure 4 shows a strong correlation between $\sigma_{d_{\mathrm{seq}}}$ and $\alpha$. However, a Kruskal-Wallis test exhibits a slight but significant difference on $\sigma_{d_{\text {seq }}} / \alpha$ between IVC_SD and SAVAM and between IVC_SD and IVC_UHD $(\mathrm{p}<0.01)$.

Results on dispersion clearly indicate that observers scan a wider visual angle when stimulated visual angle increases. Nevertheless, the fact that the ratio between $\overline{d_{\text {seq }}}$ and $\alpha$ remains constant, suggests that, until a stimulated visual angle up to $60^{\circ}$, observers scan the same proportion of the image, reaching the same salient region. The slight increase of

\begin{tabular}{cccc}
\hline & IVC_SD & SAVAM & IVC_UHD \\
\hline Optimal width & 3.9 & 9.0 & 17.7 \\
Optimal width / $\alpha$ & 0.33 & 0.30 & 0.29 \\
\hline KLD (width $=\alpha / 3)$ & 0.99 & 1.74 & 2.23 \\
Cp (width $=\alpha / 3)$ & 0.64 & 0.49 & 0.52 \\
\hline
\end{tabular}

Table 2. Optimal center model.

dispersion from SD to HD and UHD can be explained by a higher inter-observer variability due to an extended freedom of scanpath or a methodology bias due to the difference of sequence length through the datasets.

\subsection{Comparison with center models}

From the KLD and the Pearson correlation between center models of different width and saliency maps, we compute the optimal width of the center model for each dataset. It corresponds to the mean of the width that minimizes KLD and the width that maximizes Pearson correlation coefficient $\mathrm{Cp}$. Table 2 shows that optimal width increases along with stimulated visual angle but the ratio between the optimal width and $\alpha$ also remains nearly constant around $\alpha / 3$.

These results show a linear rule between optimal center model and stimulated visual angle. It confirms the previous assertion that gaze data distribution in video remains relatively stable between SD, HD and UHD viewing conditions. Moreover, it suggests that central bias is largely due to video content rather than motor bias. The optimal width of the center model, $\alpha / 3$, might reflect the rule of thirds in image and video composition.

\section{IMPROVEMENT OF VISUAL SALIENCY MODELS WITH AN OPTIMAL CENTER MODEL}

In this section, we develop a method to make visual saliency models more robust toward viewing conditions, by using the fact that deployment of visual attention is proportional to stimulated visual angle.

\subsection{Proposed method}

Some authors show that modulating visual saliency models with a center model enables to simulate central bias improving model performance[10, 12, 14]. However, the size of center models is rarely motivated. From the results in the previous section, we propose to use viewing conditions and more precisely stimulated visual angle to compute an optimal center model. The FWHM of the gaussian in the optimal center model is set as $w_{\mathrm{opt}}=\alpha / 3$. Then we can deduce $\sigma_{\mathrm{opt}}$ as:

$$
\sigma_{\mathrm{opt}}=\frac{w_{\mathrm{opt}}}{2 \sqrt{2 \ln 2}}, \text { thus } \sigma_{\mathrm{opt}} \approx \frac{\alpha}{7.06}
$$




\begin{tabular}{|c|c|c|c|c|}
\hline & \multicolumn{2}{|c|}{ SD } & \multicolumn{2}{|c|}{ UHD } \\
\hline & KLD & $\mathrm{Cp}$ & KLD & $\mathrm{Cp}$ \\
\hline $\mathrm{C}_{\mathrm{Opt}}$ & 0.96 & 0.641 & 2.17 & 0.478 \\
\hline$\overline{\mathrm{C}}_{2}^{-}-$ & $1.96 *$ & $0.552^{-}$ & $\overline{7.35} * \overline{7}$ & $0 . \overline{143} *$ \\
\hline $\mathrm{C}_{10}$ & $2.63 *$ & 0.555 & 2.54 & 0.450 \\
\hline $\mathrm{C}_{50}$ & - & - & $3.81 *$ & 0.420 \\
\hline $\mathrm{S}_{\mathrm{C}_{\mathrm{C} \text { opt }}}$ & 1.16 & 0.544 & 2.29 & 0.459 \\
\hline $\bar{S}^{--}$ & $2.84 *$ & $0.238 *$ & $\overline{3.74} *$ & $0 . \overline{29} \bar{*}$ \\
\hline $\mathrm{S}_{\mathrm{C}_{2}}$ & $2.23 *$ & 0.492 & $7.24 *$ & $0.141 *$ \\
\hline $\mathrm{S}_{\mathrm{C}_{10}}$ & $2.17 *$ & $0.375 *$ & $2.70 *$ & 0.432 \\
\hline $\mathrm{S}_{\mathrm{C}_{50}}$ & - & - & $3.23 *$ & $0.384 *$ \\
\hline$(\mathrm{SD})_{\mathrm{C}_{\mathrm{Opt}}}$ & 1.81 & 0.514 & 3.03 & 0.413 \\
\hline$\overline{\mathrm{S}} \overline{\mathrm{D}}^{---}$ & $3.28 *$ & $0.170^{*}$ & $\overline{3} . \overline{7} \overline{7} *$ & $0.260 *$ \\
\hline$(\mathrm{SD})_{\mathrm{C}_{2}}$ & $2.59 *$ & $0.440 *$ & $7.58 *$ & $0.102 *$ \\
\hline$(\mathrm{SD})_{\mathrm{C}_{10}}$ & 1.73 & 0.451 & $3.87 *$ & 0.368 \\
\hline$(\mathrm{SD})_{\mathrm{C}_{50}}$ & - & - & 3.19 & 0.371 \\
\hline $\mathrm{S}_{\mathrm{C}_{\mathrm{Opt}}} \mathrm{D}_{\mathrm{C}_{\mathrm{Opt}}}$ & 1.17 & 0.547 & 2.48 & 0.441 \\
\hline $\mathrm{SD}$ & $3.28 *$ & $0.170 *$ & $3.77 *$ & $0.260 *$ \\
\hline $\mathrm{S}_{\mathrm{C}_{2}} \mathrm{D}_{\mathrm{C}_{2}}$ & $2.59 *$ & 0.440 & $7.14 *$ & $0.120 *$ \\
\hline $\mathrm{S}_{\mathrm{C}_{10}} \mathrm{D}_{\mathrm{C}_{10}}$ & $2.46 *$ & $0.354 *$ & $2.98 *$ & 0.409 \\
\hline $\mathrm{S}_{\mathrm{C}_{50}} \mathrm{D}_{\mathrm{C}_{50}}$ & - & - & $3.17 *$ & 0.374 \\
\hline
\end{tabular}

Table 3. Performance of visual attention models with different center models. $C_{i}$ represents a center model of width $i^{\circ}$ and $\mathrm{X}_{\mathrm{C}_{\mathrm{i}}}$ represents the $\mathrm{X}$ map modulated with the $\mathrm{C}_{\mathrm{i}}$ center model. $S$ refers to static map and $D$ to dynamic map. Results marked with * are significantly different from the optimal version of the model (non-parametric test).

To assess the optimality of the proposed center model, we confront the performance of visual saliency models with the original saliency map by computing KLD and Cp as described in Section 2.3.2 for 25 videos of the IVC_SD and IVC_UHD datasets. More precisely, we compare different center, static and dynamic map fusions from the model proposed in [15]. This computational model is a bottom-up visual attention model. The fusion between static and dynamic maps is based on the maximum of the static map $a$ and the skewness of the dynamic map $b[16]$.

$$
M_{s d}=a M_{s}+b M_{d}+a b M_{s} \times M_{d}
$$

The modulation of the fusion with center model is conducted in two configurations, whether the center model is applied before or after the static-dynamic fusion.

\subsection{Results}

Results in Table 3 show that the proposed center model is always the best predictor in SD and UHD conditions. Most of the time, it significantly outperforms the other center models. The comparison of the two fusion configurations suggests that it is better to modulate maps with central model before fusion. In this case, this simple adaptation permits to improve model performance of more than $100 \%$ in SD and around 50\% in UHD. All the models (center, static and fusion) obtained better results in SD than in UHD which is consistent with results of [4] obtained on static images. In this section, we proved that an optimal center model, directly dependent on stimulated visual angle, permits to significantly improve performance of visual saliency models on professional videos. However, other improvements are required to better fit visual attention models to UHD resolution.

\section{CONCLUSION}

In this paper, we assessed the impact of visual angle on visual attention deployment. By comparing results on three eye tracking datasets on SD, HD and UHD videos, we showed that the dispersion of gaze points is directly correlated with stimulated visual angle. Results suggest that visual deployment in the video content remains relatively stable until a stimulated visual angle of about $60^{\circ}$. Moreover, we proved that an optimal center model, with a width equal to one third of stimulated visual angle, is the best predictor of visual saliency on professional videos. These results have been successfully applied to make visual saliency models more robust toward viewing conditions by modulating them with this optimal center model.

\section{ACKNOWLEDGMENT}

The authors wish to thank Josselin Rousseau for his help in generating the eye tracking UHD dataset used in this paper. This work has been supported by the UHD4U project financed by the CATRENE program.

\section{REFERENCES}

[1] ITU, "ITU-R BT.2020. Parameter values for ultra-high definition television systems for production and international programme exchange," Tech. Rep., 2012.

[2] ITU, "ITU-R BT.1769. Parameter values for an expanded hierarchy of LSDI image formats for production and international programme exchange," Tech. Rep., International Telecommunication Union, 2008.

[3] Hiromi Nemoto, Philippe Hanhart, Pavel Korshunov, and Touradj Ebrahimi, "Ultra-eye: UHD and HD images eye tracking dataset," in 2014 Sixth International Workshop on Quality of Multimedia Experience (QoMEX). sep 2014, pp. 39-40, IEEE. 
[4] Hiromi Nemoto, Philippe Hanhart, Pavel Korshunov, and Touradj Ebrahimi, "Impact of Ultra High Definition on Visual Attention," in Proceedings of the ACM International Conference on Multimedia - MM '14, New York, New York, USA, 2014, pp. 247-256, ACM Press.

[5] Duo Li, Guangtao Zhai, and Xiaokang Yang, "Ultra high definition video saliency database," in 2014 IEEE Visual Communications and Image Processing Conference. dec 2014, pp. 97-100, IEEE.

[6] Fadi Boulos, Wei Chen, Benoit Parrein, and Patrick Le Callet, "Region-of-Interest intra prediction for H.264/AVC error resilience," in 2009 16th IEEE International Conference on Image Processing (ICIP). nov 2009, pp. 3109-3112, IEEE.

[7] Yury Gitman, Mikhail Erofeev, Dmitriy Vatolin, Bolshakov Andrey, and Fedorov Alexey, "Semiautomatic visual-attention modeling and its application to video compression," in 2014 IEEE International Conference on Image Processing (ICIP). oct 2014, pp. 1105-1109, IEEE.

[8] Stefan Winkler and Ramanathan Subramanian, "Overview of Eye tracking Datasets," in 2013 Fifth International Workshop on Quality of Multimedia Experience (QoMEX). jul 2013, pp. 212-217, IEEE.

[9] ITU, "RECOMMENDATION ITU-R BT.500-11 Methodology for the subjective assessment of the quality of television pictures," Tech. Rep., 2002.

[10] Sophie Marat, Anis Rahman, Denis Pellerin, Nathalie Guyader, and Dominique Houzet, "Improving Visual Saliency by Adding 'Face Feature Map' and 'Center Bias',' Cognitive Computation, vol. 5, no. 1, pp. 6375, 2013.

[11] Olivier Le Meur and Thierry Baccino, "Methods for comparing scanpaths and saliency maps: strengths and weaknesses.," Behavior research methods, vol. 45, no. 1, pp. 251-266, 2013.

[12] Olivier Le Meur, Patrick Le, and Dominique Barba, "Predicting visual fixations on video based on low-level visual features," 2007.

[13] P. H. Tseng, Ran Carmi, Ian G M Cameron, Douglas P Munoz, and Laurent Itti, "Quantifying center bias of observers in free viewing of dynamic natural scenes," Journal of Vision, vol. 9, no. 7, pp. 4-4, jul 2009.

[14] Tilke Judd, Krista Ehinger, Fredo Durand, and Antonio Torralba, "Learning to predict where humans look," in 2009 IEEE 12th International Conference on Computer Vision. sep 2009, pp. 2106-2113, IEEE.
[15] Sophie Marat, Tien Ho Phuoc, Lionel Granjon, Nathalie Guyader, Denis Pellerin, and Anne Guérin-Dugué, "Modelling spatio-temporal saliency to predict gaze direction for short videos," International Journal of Computer Vision, vol. 82, no. 3, pp. 231-243, 2009.

[16] Satya M Muddamsetty, Desire Sidibe, Alain Tremeau, and Fabrice Meriaudeau, "A performance evaluation of fusion techniques for spatio-temporal saliency detection in dynamic scenes," in 2013 IEEE International Conference on Image Processing. sep 2013, pp. 3924-3928, IEEE. 